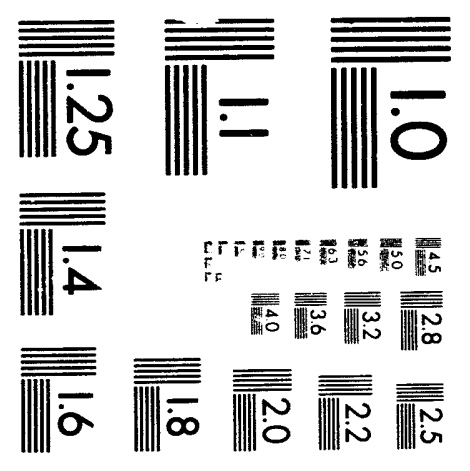



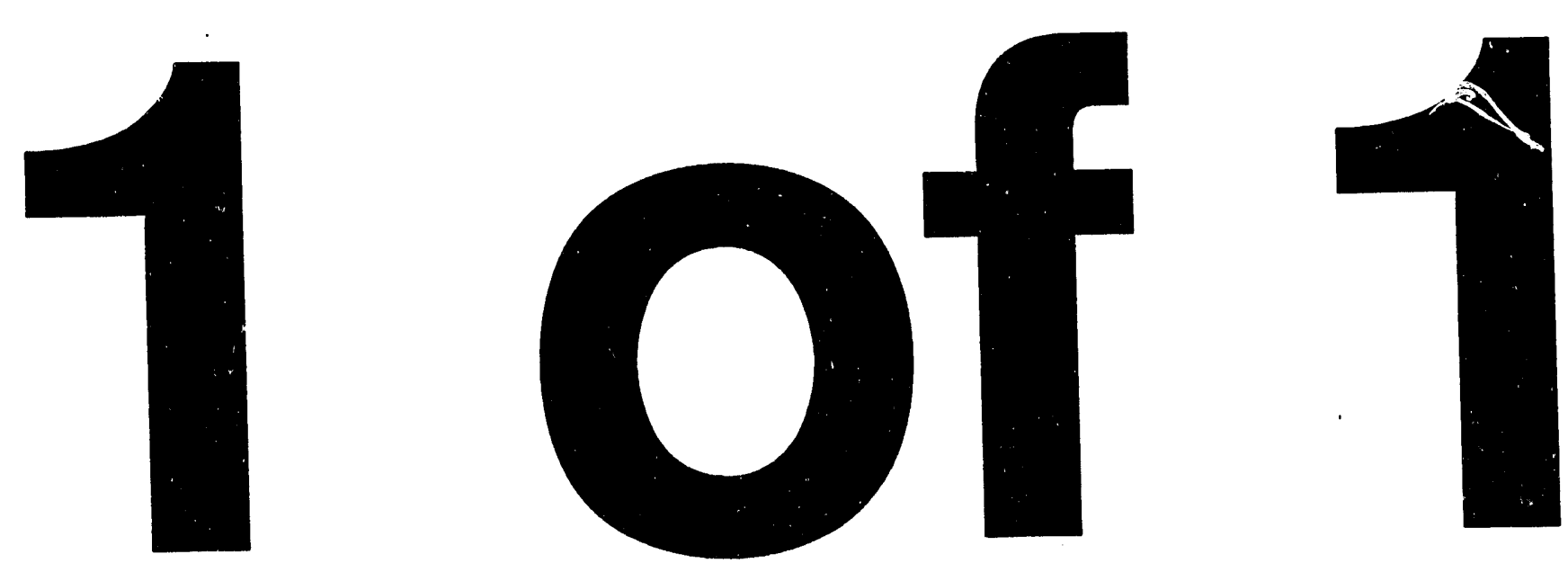


\title{
Full Cycle Beam Diagnostics with an Ionization Profile Monitor*
}

\author{
Arnold Stillman and R. E. Thern \\ Brookhaven National Laboratory, Upton, NY 11973
}

May 14, 1993

\begin{abstract}
The Alternating Gradient Synchrotron Booster at Brookhaven National Laboratory uses an ionization profile monitor to generate profiles of proton and heavy-ion beams. The profile monitor can acquire hundreds of profiles during an acceleration cycle, and then display aud store them for analysis. Profiles appear in real time on an oscilloscope-type display, but other visualizations are available as well, namely mountain range and emittance displays. File storage of profile data is simple, as is the storage of moments and emittances.
\end{abstract}

\section{INTRODUCTION}

The Alternating Gradient Synchrotron (AGS) Booster at Brookhaven is both a proton accumulator and $a$ heavy ion accelerator. The heavy-ion requirement of an ultra-high vacuum implies an ionization device with very high gain. The AGS Booster uses an ionization profile monitor (IPM) composed of a microchannel plate and multi-anode readout. A separate paper describes the profile monitor in detail [1]. This report is essentially a description of the user interface.

\section{THE USER INTERFACE}

The IPM can display profiles in real time at any particular trigger time during the cycle or show statistics from the profiles of a complete cycle, all at a glance and immediately after the acquisition of the final profile. Also useful and quite striking is the movie

-Work performed under the auspices of the U.S. Department of Energy. mode, in which a sequence of profiles appears as fast as the program can display them. The apparent time in the movie is about a thousand times slower than real time. The movie display also makes great use of the magnet cycle. Correlations of beam motion and energy are clearly visible, since the magnet cycle appears in a contrasting color, with a moving dot indicating the displayed profile's occurence in the cycle.

Several figures should serve to illustrate these points. The basic display in Figure 1 shows the horizontal and vertical profiles of a typical proton beam at $72.58 \mathrm{~ms}$ after injection. The time displayed in the center between the two profiles is the time during the cycle, but injection occurs at about $40 \mathrm{~ms}$., so this set of profiles represents the beam at about $33 \mathrm{~ms}$ into the acceleration cycle. Below the graphical display is a collection of numerical data, which includes integration time, trigger time, statistical moments, raw amplitude minima and maxima, and various instrument readbacks. The most important numbers are the three moments of the Gaussian fit to the profile: M0, the total area,; M1, the centroid in mm.; M2, the $1 \sigma$ width.

Obviously, we can not illustrate the movie display. However, some "frames" from a typical movie can give an idea of the display. In Figure 2, three separate frames show $\mathrm{Au}^{+33}$ profiles and the magnet cycle. An extraction bump is causing the beam to move radially outward. Some qualitative differences in beam motion are apparent on this time scale, but synchrotron motion is too rapid to resolve, even at our fastest scan rate.

More typical for accelerator operators is a mountain range display. Figure 3 shows a typical mountain range display, using proton profiles. Compare the motion in this figure with that of the motion in Figure 2.

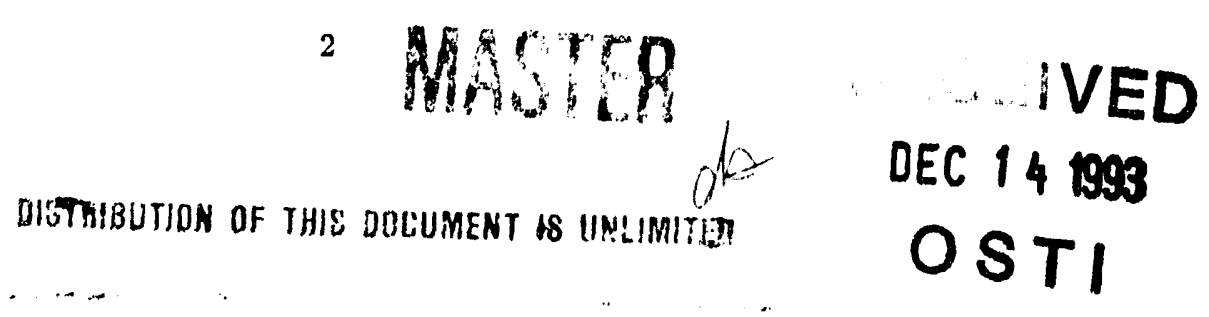




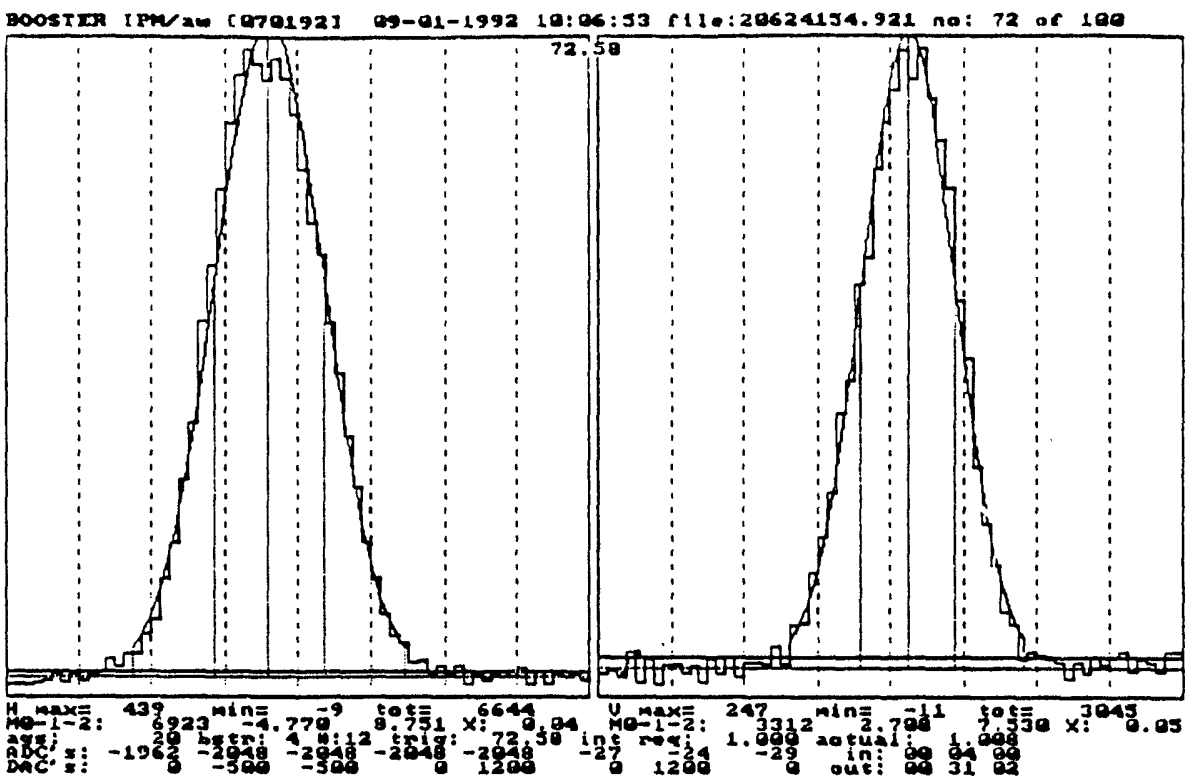

Figure 1: The Profile Display. Typical profiles of the proton berm in the AGS Booster. On the left is the horizontal profile and on the right, the vertical. The channel spacing is $1.4 \mathrm{~mm}$ and the vertical dashed lines occur every eight channels. Radially inward is to the right in the horizontal profile, and vertically upward is to the right in the vertical profile.

Finally, perhaps the most informative of the available modes is one in which the normalized emittance appears as a function of time in the cycle. In conjunction with the magnet cycle display, studies of emittance growth are now on-line operations. The definition of normalized emittance ultimately relies on the magnet cycle to determine $\beta$ and $\gamma(\beta$ and $\gamma$ are the familiar relativistic $v / c$ and $\left.1 / \sqrt{1-v^{2} / c^{2}}\right)$. The specified error between the magnet programming function and the actual magnetic field in the magnets is on the order of $5 \%[2]$.

\section{NULI, SUBTRACTION}

The AGS Booster has a mode of operation in which four short cycles of proton acceleration, preceded by a cycle with no beam, define a larger cycle, known in this case as User 1 . User 1 provides a handy way to do null subtraction. First, however, we provide a description of the User structure of the Booster magnet cycle. There are up to four separate magnet cycles that are available during Booster operations. Each one is a User, in the sense that it serves a particular physics or beam studies user. A complete set of Users make up a Booster "supercycle." Although the number of Users is variable from one to four, anyone operating a beam device, such as the IPM, only sees the display of the selected User. The timing selection software ignores unwanted Users.

To do null subtraction, we take advantage of the fact that the first pulse of User 1 is "empty" in the sense that the magnet cycle is present but there is no injected beam. This generates a pulse with all the systematic noise signals of the actual acceleration $c y-$ cle, but none of the beam-induced signals. The IPM display program uses this empty pulse to generate null levels. Subtracting these null leveis from the actual profiles produces extraordinarily clea a siga. data. For example, Figure 4 shows the san a urc e with the systematic background presen ari $a b$. $t$.

\section{References}

[1] A. N. Stillman, R. Thern, and R. L. Wove: Rev. Sci. Instrum. 63, 3412 (1992)

[2] A. Soukas, private communication. 

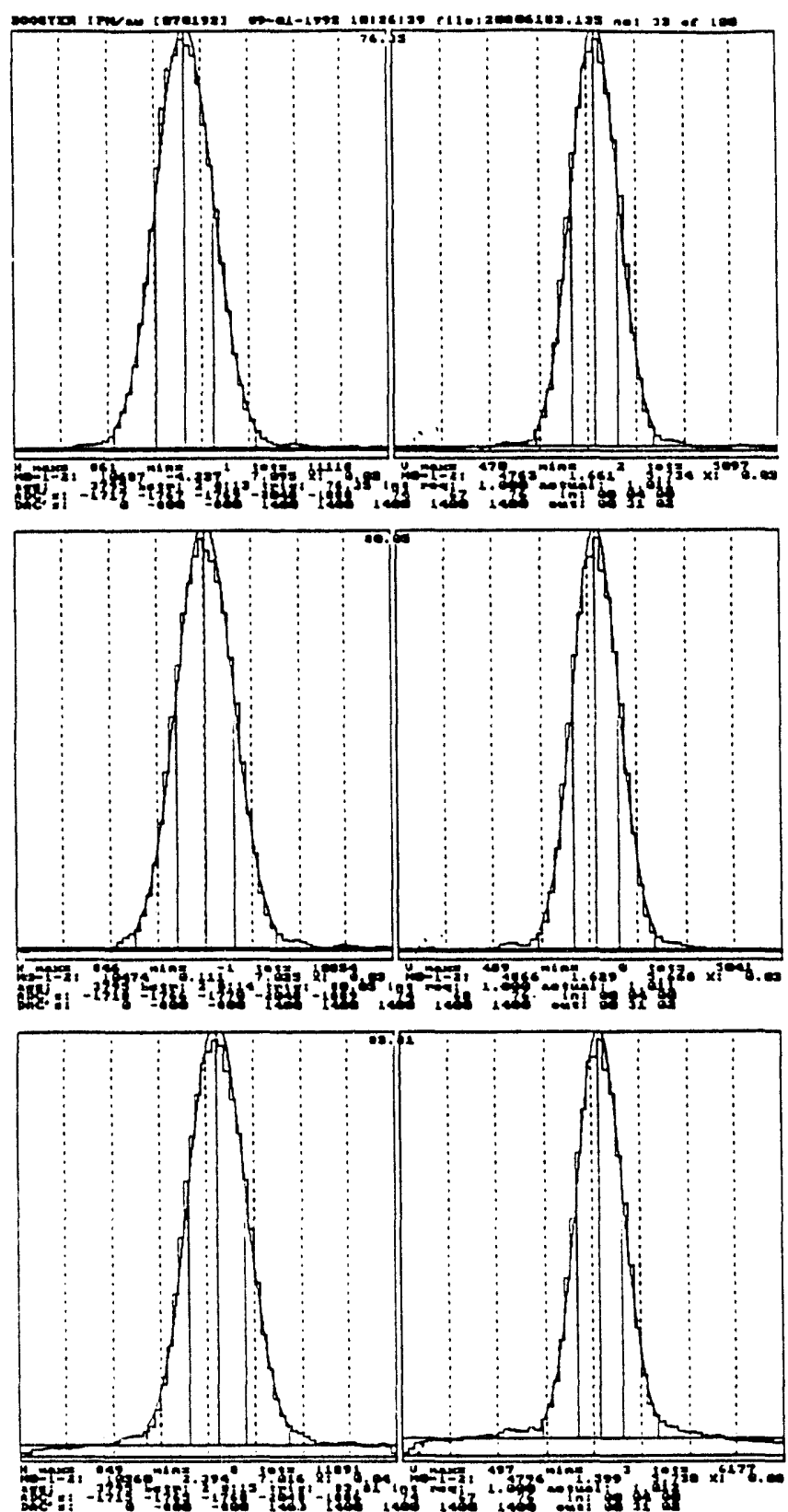

Figure 2: The Movie. Three frames from a movie of all the profiles in a full acceleration cycle. The display is of the same beam as in Figure 2. The spacing between profiles is about $3.5 \mathrm{~ms}$.

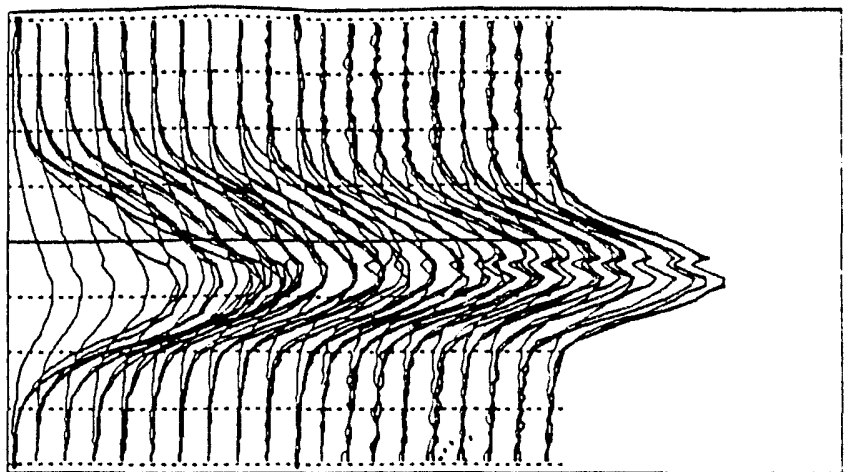

Figure 3: The Mountain Range. A typical mountain range display of the horizontal profiles of the beam in Figure 2. The time axis extends through the entire cycle.

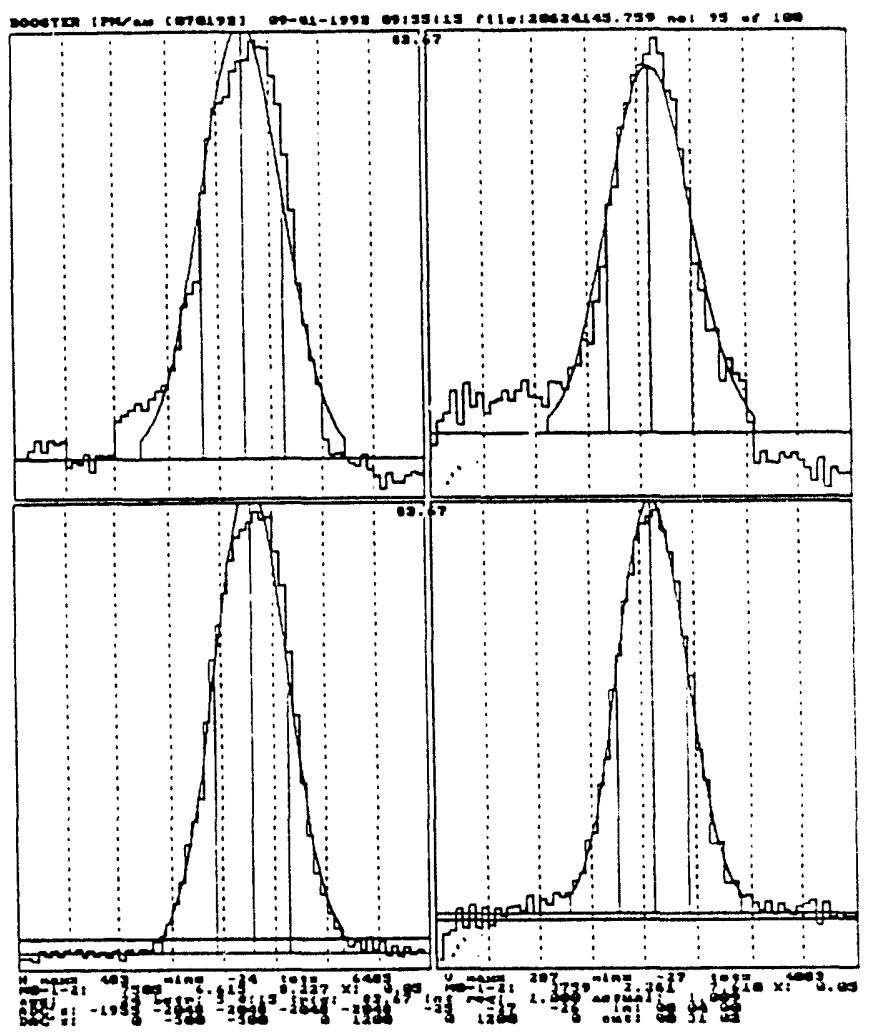

Figure 4: Null Subtraction a.A typical proton profile superimposed upon systematic noise. b. The same profile, but after removal of systematic noise with presampled null values. The sampling of the null values occurs in an empty pulse, but at the same time in the pulse as the sampling of subsequent profile data. 


\section{DISCLAIMER}

This report was prepared as an account of work sponsored by an agency of the United States Government. Neither the United States Government nor any agency thereof, nor any of their employees, makes any warranty, express or implied, or assumes any legal liability or responsibility for the accuracy, completeness, or usefulness of any information, apparatus, product, or process disclosed, or represents that its use would not infringe privately owned rights. Reference herein to any specific commercial product, process, or service by trade name, trademark, manufacturer, or otherwise does not necessarily constitute or imply its endorsement, recommendation, or favoring by the United States Government or any agency thereof. The views and opinions of authors expressed herein do not necessarily state or reflect those of the United States Government or any agency thereof. 

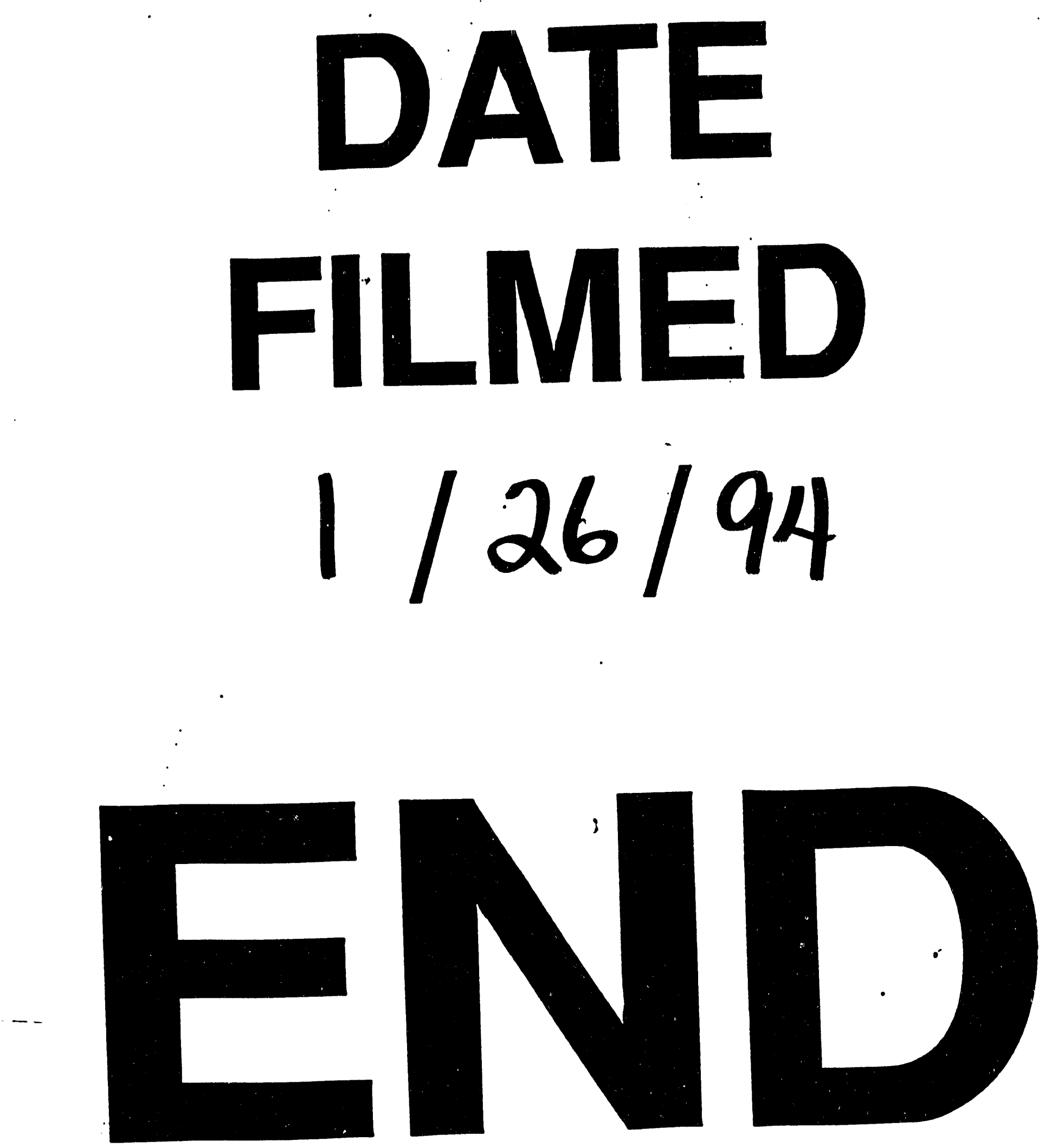


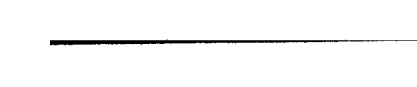

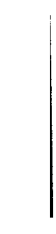

